ORIGINAL RESEARCH

\title{
Predictive Factors for Determining the Clinical Severity of Pediatric Scorpion Envenomation Cases in Southeastern Turkey
}

\author{
Aykut Çağlar, MD; Halil Köse, MD; Aslan Babayiğit, MD; Taliha Öner, MD; Murat Duman, MD \\ From the Department of Pediatric Emergency Care, Dokuz, Eylul University Faculty of Medicine, Izmir, Turkey (Drs Çă̆lar and Duman); the \\ Department of Pediatrics, Şanlıurfa Children's Hospital, Şanlıurfa, Turkey (Drs Babayiğit and Köse); and the Division of Pediatric Cardiology, \\ Department of Pediatrics, Şanluurfa Children's Hospital, Şanlıurfa, Turkey (Dr Öner).
}

\begin{abstract}
Objective.-The aim of this study was to define the epidemiological, clinical, and laboratory manifestations of scorpion envenomation and to identify factors that are predictive of severe cases.

Methods. - The medical files of 41 scorpion envenomation cases were reviewed retrospectively. The cases were classified as mild-moderate or severe. The epidemiological, clinical, and laboratory findings of patients were recorded.

Results.-There were 27 patients (65.9\%) in the mild-moderate group and 14 patients $(34.1 \%)$ in the severe group. The median age of all patients was 48 months. The most common systemic finding was cold extremities (41.5\%). In all patients, the most commonly observed dysrhythmia was sinus tachycardia $(34.1 \%)$. Two patients $(4.9 \%)$ had pulseless ventricular tachycardia and died. Pulmonary edema and myocarditis were observed in 9 patients (22\%). Median values of leukocyte and glucose levels were markedly increased in the severe group. Additionally, the mean thrombocyte level $(540,857$ $\pm 115,261$ cells $\left./ \mathrm{mm}^{3}\right)$ in the severe group was significantly increased compared with the mild-moderate group $\left(391,365 \pm 150,017\right.$ cells $\left./ \mathrm{mm}^{3}\right)$. Thrombocyte levels exhibited a positive correlation with leukocyte and glucose values and a negative correlation with patient left ventricular ejection fraction. Multivariate analysis of laboratory parameters indicated that the most predictive factor for clinical severity is thrombocytosis (odds ratio 23.9 ; 95\% CI: $1.6-353.5, P=.021$ ).

Conclusions.-Although our results share some similarities with those of other reports, thrombocytosis was markedly increased in the severe group and served as the most predictive laboratory factor of clinical severity.
\end{abstract}

Key words: scorpion, pediatric, thrombocytosis, ventricular tachycardia, critical care

\section{Introduction}

Scorpion envenomation is a life-threatening condition in different regions of the world, particularly for pediatric populations. The condition is an endemic problem in the Middle East, South America, India, and Africa. In southeastern and eastern Turkey, scorpion envenomation affects hundreds of people every year. ${ }^{1}$ Sanliurfa is one of the cities in Turkey that has a high incidence of scorpion envenomation. ${ }^{2}$ In the world, 1500 of species of scorpions in 18 families have been described. ${ }^{3}$ With 25 of these species, envenomation can cause medical problems leading to various clinical conditions, some

Corresponding author: Murat Duman, MD, Dokuz Eylul University Medical Faculty, Department of Pediatric Emergency Care, İzmir, Turkey (e-mail: mduman@deu.edu.tr). of which may result in death. In Turkey, especially in the southeastern region, species such as Androctonus crassicauda and Leiurus quinquestratus are responsible for severe cases and mortality. ${ }^{2}$

The majority of patients affected by scorpion envenomation have mild symptoms, including localized pain. ${ }^{4}$ The lower the body weight and age of the victim, the more serious scorpion envenomation becomes. Therefore, children exhibit more severe symptoms and have a considerably increased mortality rate compared with adults. ${ }^{5}$ Other factors that affect clinical severity of envenomation include scorpion species and size as well as the health status of the victim. Activation of the sympathetic and parasympathetic nervous systems is the main cause of severe clinical manifestations and death., The condition referred to as "autonomic storm" may 
cause pulmonary edema or cardiopulmonary failure. ${ }^{8}$ In endemic regions, medical awareness regarding scorpion envenomation is crucial. All practitioners should recognize the severe clinical manifestations of a scorpion sting. Preventing mortality in these cases depends on early intervention and remarkably good critical care.

The aim of this study was to define the epidemiological, clinical, and laboratory manifestations of scorpion envenomation. An additional aim was to characterize factors predictive of a poor prognosis.

\section{Methods}

\section{PATIENTS}

All scorpion envenomation cases admitted to the emergency department at Sanliurfa Children's Hospital over a 2-year period (2011-2012) were retrospectively included in this study. All children in this study were less than 18 years of age. The diagnosis was based on patient history and hospital medical records.

\section{METHODS}

Patients' medical records were reviewed retrospectively for epidemiological, clinical, and laboratory findings. Epidemiological data included age, sex, body weight, health status of victim, sting location on the body, where and when the sting occurred, and time to admission to the emergency department. Clinical data such as fever, vomiting, sweating, salivation, priapism, mydriasis, myocarditis, and pulmonary edema were also collected. Vital signs (tachypnea, bradycardia, tachycardia, hypotension, and hypertension) were defined according to normal values for age groups. ${ }^{9}$ Myocarditis was diagnosed if the patient had clinical findings of heart failure (tachycardia, gallop rhythm, muffled heart sounds, or a new murmur) and at least 1 of the following: electrocardiogram changes, cardiac dysfunction on echocardiography, or increased troponin levels. ${ }^{10}$ Duration of stay in the intensive care unit, mechanical ventilation time, intubation time, and all administered treatments (scorpion antivenom, doxazosin, inotropic, and vasoactive drugs) were recorded for each patient. Laboratory values included white blood cell and hemoglobin concentrations, platelet count, blood glucose, blood urea, creatine, aspartate aminotransferase, alanine aminotransferase, creatine phosphokinase (CPK), creatine kinase-myocardial band fraction (CK-MB), troponin I, lactate, and C-reactive protein. This study was approved by local ethical committee of Harran University (Sanliurfa, Turkey).

The patients were grouped according to a grading system (Table 1): the severe grade group included patients with severe clinical findings, such as acute pulmonary edema,
Table 1. Grading system according to clinical findings

\begin{tabular}{ll}
\hline Mild-moderate grade & \multicolumn{1}{c}{ Severe grade } \\
\hline Asymptomatic patients & Severe breathing difficulties \\
Local pain & Nystagmus \\
Sialorrhea & Lethargy, stupor, coma \\
Dysphagia & Focal/generalized seizures \\
Irritability & Increased muscular tone \\
Tachycardia & Low blood pressure \\
Tachypnea & Cardiac failure \\
Mild and moderate & Acute pulmonary edema \\
breathing difficulties & \\
\hline
\end{tabular}

myocarditis, cardiopulmonary failure, shock, and coma; the mild-moderate grade group included asymptomatic patients and patients with local or mild systemic findings.?

\section{STATISTICAL ANALYSIS}

The SPSS software, version 15 (SPSS, Inc., Chicago, IL), was used to analyze data. Quantitative data are described as mean $\pm \mathrm{SD}$ or median with interquartile range (IQR), if data followed nonnormal distribution. The Mann-Whitney $U$ test and $\chi^{2}$ test were used to compare the 2 groups (mild-moderate and severe). Risk factors were evaluated in multivariate analyses using a multiple logistic stepwise regression procedure. The Pearson correlation coefficient was used to assess the relationship between laboratory values and clinical severity. Probabilities ( $P$ values) less than 0.05 were considered significant for all tests.

\section{Results}

The records of 41 patients who were stung by a scorpion were analyzed. The epidemiologic and demographic characteristics of the patients are summarized in Table 2. There were 27 patients $(65.9 \%)$ in the mildmoderate group and 14 patients $(34.1 \%)$ in the severe group. The majority of stings in both groups (36.6\%) occurred in July. The median age of the person stung was 48 months (IQR: 36-96); the median age of the severe group was significantly less than that of the mildmoderate group $(P=.013)$. The majority of cases $(58.5 \%)$ were boys. The median body weight of all patients was $16 \mathrm{~kg}$ (IQR: 13.5-30); the severe group had lower body weight than the mild-moderate group $(P=$ .044). The median duration between sting and hospital admission was longer for the severe group compared with the mild-moderate group $(P<.001)$.

The clinical manifestations of scorpion sting are summarized in Table 3. The most common finding was pain; the most common systemic clinical findings were 
Table 2. Epidemiologic and demographic parameters of scorpion sting victims

\begin{tabular}{|c|c|c|c|c|}
\hline & Total & Mild-moderate grade & Severe grade & \\
\hline Parameters & $n=41(100 \%)$ & $n=27(65.9 \%)$ & $n=14(34.1 \%)$ & $P$ value ${ }^{a}$ \\
\hline Age, months & $48.0(36-96)$ & $83(38-127)$ & $42(26.5-52.7)$ & .013 \\
\hline Body weight, kg & $16.0(13.5-30)$ & $23(14-33)$ & $15(12.7-15.2)$ & .044 \\
\hline Sex & & & & .189 \\
\hline Male & $24(58.5)$ & $18(66.7)$ & $6(42.9)$ & \\
\hline Female & $17(41.5)$ & $9(33.3)$ & $8(57.1)$ & \\
\hline Admission time & & & & .035 \\
\hline Day & $8(19.5)$ & $8(29.6)$ & $0(0)$ & \\
\hline Night & $33(80.5)$ & $19(70.4)$ & $14(100)$ & \\
\hline Sting site & & & & .68 \\
\hline Upper extremity & $27(65.9)$ & $17(63)$ & $10(71.4)$ & \\
\hline Lower extremity & $12(29.3)$ & $9(33.3)$ & $3(21.4)$ & \\
\hline Head and neck & $2(4.9)$ & $1(3.7)$ & $1(7.1)$ & \\
\hline Time to admission, $\mathrm{h}$ & $2(1-3)$ & $1(1-2)$ & $3(2-4.5)$ & $<.001$ \\
\hline Hospital stay, h & $48(24-102)$ & $36(24-48)$ & $172(90-222)$ & $<.001$ \\
\hline Outcome & & & & .111 \\
\hline Dead & $2(4.9)$ & $0(0)$ & $2(14.3)$ & \\
\hline Alive & $39(95.1)$ & $27(100)$ & $12(85.7)$ & \\
\hline
\end{tabular}

*Values are median (interquartile range) or $n(\%)$.

${ }^{a}$ Mann-Whitney $U$ test and $\chi^{2}$ test were used to compare the 2 groups.

Table 3. Clinical manifestations of scorpion sting victims

\begin{tabular}{|c|c|c|c|c|}
\hline & Total & Mild-moderate grade & Severe grade & \\
\hline Manifestation & $n=41(100 \%)$ & $n=27(65.9 \%)$ & $n=14(34.1 \%)$ & $P$ value ${ }^{a}$ \\
\hline Pain & $39(95.1)$ & $25(92.6)$ & $14(100)$ & .539 \\
\hline Vomiting & $15(36.6)$ & $4(14.8)$ & $11(78.5)$ & $<.001$ \\
\hline Salivation & $13(31.7)$ & $3(11.1)$ & $10(71.4)$ & $<.001$ \\
\hline Sweating & $14(34.1)$ & $3(11.1)$ & $11(78.5)$ & $<.001$ \\
\hline Priapism $^{b}$ & $1(4)$ & $0(0)$ & $1(16.6)$ & .341 \\
\hline Fever & $7(17.1)$ & $2(7.4)$ & $5(35.7)$ & .35 \\
\hline Tachypnea & $16(39)$ & $2(7.4)$ & $14(100)$ & $<.001$ \\
\hline Blood pressure & & & & $<.001$ \\
\hline Normal & $23(56.1)$ & $21(77.8)$ & $2(14.3)$ & \\
\hline Hypertension & $15(36.6)$ & $6(22.2)$ & $9(64.3)$ & \\
\hline Hypotension & $3(7.3)$ & $0(0)$ & $3(21.4)$ & \\
\hline Cold extremity & $17(41.5)$ & $6(22.2)$ & $11(78.6)$ & .001 \\
\hline Tachycardia & $14(34.1)$ & $2(7.4)$ & $12(85.7)$ & $<.001$ \\
\hline Bradycardia & $1(2.4)$ & $1(3.7)$ & $0(0)$ & \\
\hline VT & $2(4.9)$ & $0(0)$ & $2(14.3)$ & .11 \\
\hline Murmur & $6(14.6)$ & $0(0)$ & $6(42.9)$ & .001 \\
\hline Pulmonary edema & $9(22)$ & $0(0)$ & $9(64.3)$ & $<.001$ \\
\hline Myocarditis & $9(22)$ & $0(0)$ & $9(64.3)$ & $<.001$ \\
\hline Mydriasis & $2(4.9)$ & $0(0)$ & $2(14.3)$ & .11 \\
\hline Agitation & $14(34.1)$ & $2(7.4)$ & $12(85.7)$ & $<.001$ \\
\hline Seizure & $1(2.4)$ & $0(0)$ & $1(7.1)$ & .341 \\
\hline
\end{tabular}

Values are $n(\%)$.

${ }^{a}$ Male patients.

${ }^{b}$ The $\chi^{2}$ test was used to compare the 2 groups.

VT, ventricular tachycardia. 
cold extremities, tachypnea, vomiting, sweating, tachycardia, and agitation. All of these systemic findings were increased in the severe group, and the differences from the mild-moderate group were statistically significant. Fever was noted in 7 patients $(17.1 \%)$. In the severe group, 5 patients had fever, and 2 of these patients had mydriasis. One of the patients with mydriasis had a seizure. Both patients with mydriasis died. Myocarditis was frequently observed in patients with fevers, a finding that was statistically significant $(P=.03)$. The most commonly observed dysrhythmia in all patients was sinus tachycardia, observed in 14 patients (34.1\%). Two patients $(4.9 \%)$ had pulseless ventricular tachycardia, and both died. Pulmonary edema and myocarditis were observed in 9 patients (22\%). Neurologic manifestations such as agitation, lethargy, convulsion, and mydriasis were observed in 14 patients $(34.1 \%)$. Agitation was the most frequently seen neurologic manifestation. Convulsion was observed in 1 patient, and that patient was also 1 of the 2 who died.

Laboratory features of all cases are summarized in Table 4. Leukocyte and thrombocyte values and glucose levels were markedly increased in the severe group. Thrombocyte levels exhibited a positive correlation with leukocyte and glucose values and a negative correlation with left ventricular ejection fraction (Figure 1). Cardiac markers (troponin I, CPK, and CK-MB) were obtained on admission and at follow-up. With the exception of 2 patients in the severe group, troponin I levels were elevated on admission and gradually increased, reaching a peak at 24 hours. Thereafter, the levels exhibited a downward trend (Figure 2A). However, CK-MB levels were increased in the severe group on admission and then gradually decreased (Figure 2B). Multivariate analyses of laboratory parameters (leukocyte, thrombocyte, glucose, troponin, CK-MB, and lactate) revealed that the most predictive factor for clinical severity was thrombocytosis (odds ratio 23.9; 95\% CI: 1.6-353.5, $P=.021)$.

Echocardiography was performed on 35 patients (85\%), and left ventricular ejection fraction (LVEF) and fractional shortening (FS) were measured. In the severe group, the median LVEF (39.5, IQR: 33.5-49.0) and FS value (19.5, IQR: 15.7-23.0) were reduced

Table 4. Association of laboratory data and severity of envenomation

\begin{tabular}{|c|c|c|c|c|}
\hline & All patients & Mild-moderate grade & Severe grade & \\
\hline Parameters & $n=41$ & $n=27$ & $n=14$ & $P$ value $^{a}$ \\
\hline Leukocytes, $\times 10^{3} / \mathrm{mL}$ & $13,480(9060-30,375)$ & $9700(7960-13,480)$ & $32,325(20,412-37,837)$ & $<.001$ \\
\hline Hemoglobin, g/dL & $12.7 \pm 1.6$ & $12.5 \pm 1.7$ & $13.0 \pm 1.25$ & .5 \\
\hline Platelets, $\times 10^{3} / \mathrm{mL}$ & $391.365 \pm 150.017$ & $391.365 \pm 150.017$ & $540.857 \pm 115.261$ & $<.001$ \\
\hline Glucose, mg/dL & $125.3(100.4-175.8)$ & $108(92-135.2)$ & $228.6(127.8-302.3)$ & .001 \\
\hline Urea, mg/dL & $29.0 \pm 8.5$ & $26.9 \pm 7.9$ & $33.1 \pm 8.2$ & .78 \\
\hline Creatinine, $\mathrm{mg} / \mathrm{dL}$ & $0.43 \pm 0.13$ & $0.40 \pm 0.12$ & $0.50 \pm 0.14$ & .03 \\
\hline ALT, IU/L & $21.6(16.8-26.6)$ & $19.9(16.4-24)$ & 24.5 (19.9-29.6) & .108 \\
\hline AST, IU/L & $36.7(30.5-47.1)$ & $31.6(29.3-38.4)$ & $46.4(37.6-55.7)$ & .002 \\
\hline CPK, IU/L & $191.9(135-314)$ & $137.8(121.8-215)$ & $314(274-632.3)$ & $<.001$ \\
\hline \multicolumn{5}{|l|}{ CK-MB, IU/L } \\
\hline 0 hours & $40.7(31.3-83.9)$ & $36.5(29.8-40.7)$ & $101.6(75.1-134.4)$ & $<.001$ \\
\hline 12 hours & $56.0(33.3-97.8)$ & $33.3(22.5-54.3)$ & $92.2(55.7-103.7)$ & .006 \\
\hline 24 hours & $68.2(33.5-99.7)$ & $32.9(25.6-53.9)$ & $88.8(57.5-119.4)$ & .02 \\
\hline 48 hours & $52.6(35-81.8)$ & $35(28-47.4)$ & $69(44.6-117.5)$ & .236 \\
\hline 96 hours & $44.0(36.6-69.5)$ & $41.0(38.1-44)$ & $44.35(35.6-84.4)$ & .615 \\
\hline \multicolumn{5}{|l|}{ Troponin-I, ng/mL } \\
\hline 0 hours & $0.028(0.01-1.12)$ & $0.010(0-0.098)$ & $1.19(0.18-5.35)$ & $<.001$ \\
\hline 12 hours & $5.09(2.40-12.6)$ & $0.001(0.0002-1.76)$ & $9.88(3.38-15.3)$ & .004 \\
\hline 24 hours & $9.40(2.08-16.4)$ & $0.009(0.003-1.96)$ & $10.82(5.1-22.8)$ & .011 \\
\hline 48 hours & $4.25(1.9-8.8)$ & $2.37(1.38-3.36)$ & $6.2(2.02-9.16)$ & .236 \\
\hline 96 hours & $0.86(0.05-5.42)$ & $0.05(0.001-0.1)$ & $2.7(0.7-6.29)$ & .079 \\
\hline Lactate, $\mathrm{mmol} / \mathrm{L}$ & $2.5(1.3-4.1)$ & $2.0(1.3-2.7)$ & $5.4(1.3-6.4)$ & .026 \\
\hline C-reactive protein, $\mathrm{mg} / \mathrm{L}$ & $0.40(0.23-0.68)$ & $0.4(0.23-0.96)$ & $0.34(0.23-0.67)$ & .690 \\
\hline
\end{tabular}

Values are median (interquartile range) or mean $\pm \mathrm{SD}$.

${ }^{a}$ Mann-Whitney $U$ test was used to compare the 2 groups.

ALT, alanine aminotransferase; AST, aspartate aminotransferase; CPK, creatine phosphokinase; CK-MB, creatine kinase-myocardial band. 

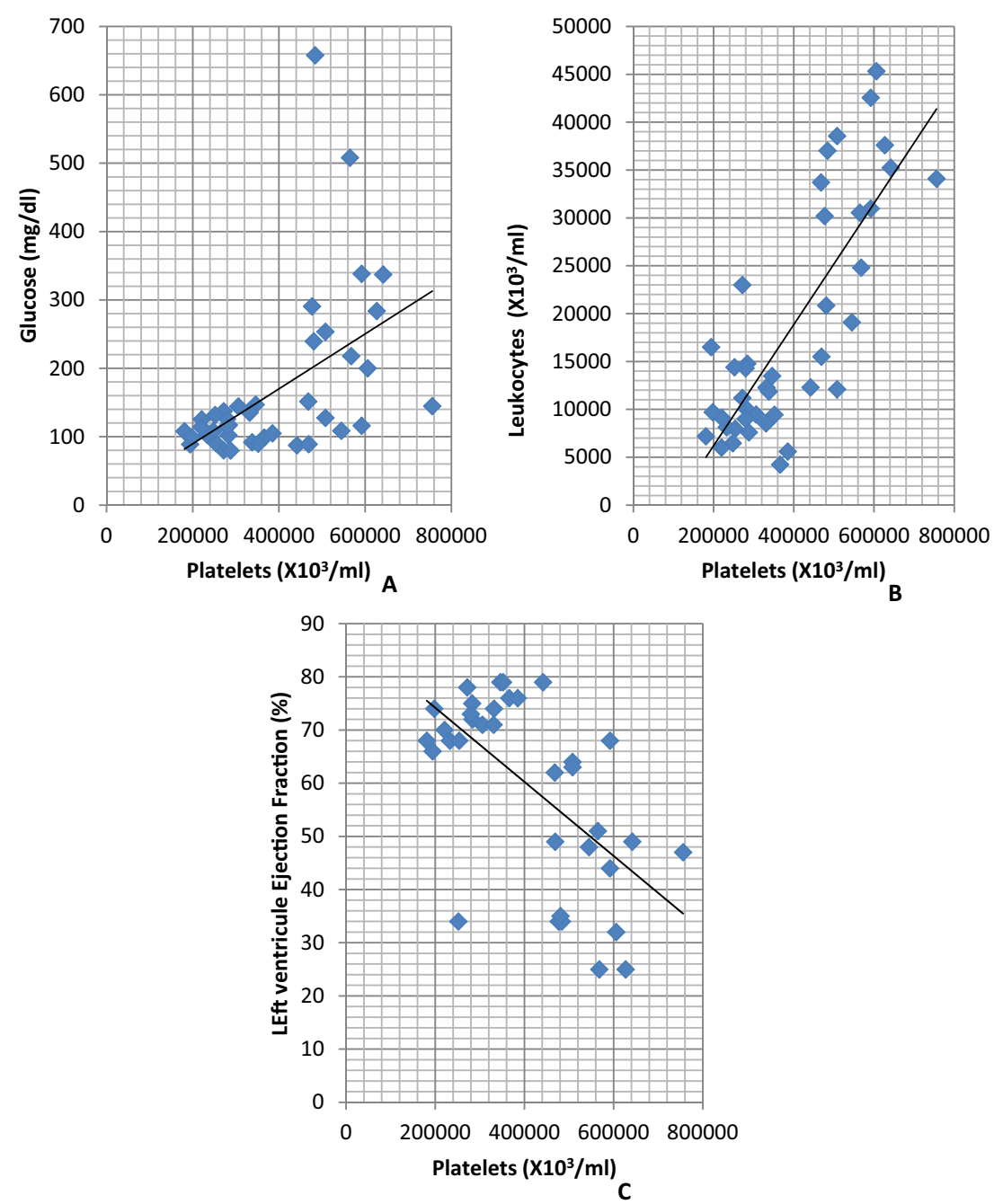

Figure 1. The correlations between platelets and glucose, leukocytes, and left ventricular ejection fraction. (A) Platelets and glucose are positively correlated $(r=.507 ; P=.001)$. (B) Platelets and leukocytes are positively correlated $(r=.795 ; P<.001)$. (C) Platelets and left ventricular ejection fraction are negatively correlated $(r=-0.613 ; P<.001)$.

compared with the mild-moderate group (LVEF 72, IQR: $68-76$, and FS 40, IQR: $36.5-41 ; P<.001$ ).

Most patients, with the exception of 2, were treated with scorpion antivenom (Refik Saydam Hygiene Center, Sihhiye, Turkey). The median duration of scorpion antivenom administration was 2 hours (IQR: 1-2). The median administration time was longer in the severe group (2.5 hours; IQR: 2.0-4.5) compared with the mild-moderate group (1.0 hours, IQR: 1.0-2.0), and the result was statistically significant $(P<.001)$. Doxazosin was administered to 9 patients $(22 \%)$ as the first option because prazosin was not available in our country. The first choice of inotropic drugs was dobutamine, and first choice of vasodilator agents was nitroglycerin. Dobutamine was administered to 15 patients (36.6\%), and nitroglycerin was administered to $6(14.6 \%)$. The median duration of inotropic drug usage was 83.0 hours (IQR: 31-96). Mechanical ventilation support was used for 10 patients (24.4\%), and the median duration was 42.0 hours (IQR: 4.7-69.0).

Two of the 41 patients $(4.9 \%)$ died. One was a 25 month-old girl who was stung by a scorpion on her left hand and brought to the emergency department within 2 hours. On admission, she was lethargic, with fever, mydriasis, hypersalivation, tachycardia, and respiratory distress. On admission to the pediatric intensive care unit, she was intubated because of pulmonary edema and myocarditis. Scorpion antivenom and doxazosin were administered immediately; dobutamine and nitroglycerin were started for inotropic and vasodilator effect. The patient also had a seizure and anisocoria for 12 hours of her stay, but cranial imaging could not be performed. 

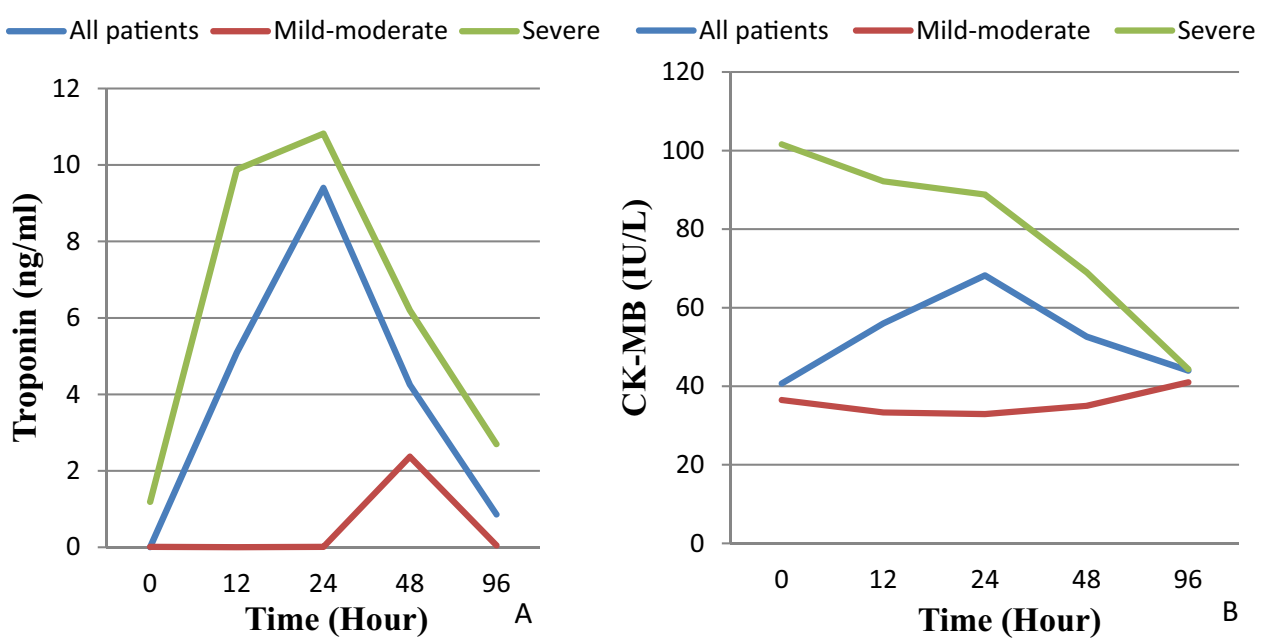

Figure 2. Median troponin and creatine kinase-myocardial band (CK-MB) levels of patients by group: all patients (blue lines), mild-moderate (red lines), and severe (green lines). (A) Troponin levels peaked at 24 hours for all groups. (B) In severe group, the CK-MB levels were high on admission and gradually decreased until follow-up.

On the second day of her stay in the pediatric intensive care unit, she had pulseless ventricular tachycardia, and electrical defibrillation was performed; she did not respond to the interventions, and died. The other patient who died, a 44-month-old girl, was stung by a scorpion on her neck. On admission, she was lethargic, and had fever, mydriasis, hypersalivation, tachycardia, and respiratory distress. She had neurological manifestations similar to those of the other patient who died, except for anisocoria and seizure, and she had pulmonary edema and myocarditis. Although respiratory and inotropic support was given immediately, she had pulseless ventricular tachycardia and died in 26 hours.

\section{Discussion}

Scorpion envenomation is endemic to Southeastern Turkey, ${ }^{5}$ and $A$ crassicauda and $L$ quinquestratus species dominate this region. Their sting carries high rates of morbidity and mortality. ${ }^{2}$ Epidemiological, clinical, and laboratory characteristics have been previously discussed. ${ }^{1,2,6,7,11,12}$ Studies from Turkey indicate that $A$ crassicauda is most often observed. Although $L$ quinquestratus is rarely observed, ${ }^{6,7}$ it is one of the most lethal scorpion species. ${ }^{13}$ Previous reports show that $L$ quinquestratus sting can have similar clinical effects compared with A crassicauda. ${ }^{14,15}$ In our study, we were unable to identify the type of scorpion involved.

In our study, males were more frequently stung than females, a finding consistent with the results of other studies. ${ }^{6,7,16,17}$ Other studies have also demonstrated that clinical symptoms are more serious in young children. ${ }^{6}$ Our patients in the severe group were younger than patients in the mild-moderate group. Moreover, patients in the severe group had lower body weight compared with the mild-moderate group, which is also consistent with the literature. ${ }^{18}$ The most common sting site involved the extremities. Only 2 patients were stung on the neck while sleeping, and 1 of them died. All of the patients lived in rural areas and had a low socioeconomic status. Most of the children typically played outside of their homes. Walking barefoot is widespread in this region; various studies have supported this finding. ${ }^{2,5,7,17}$

In the literature, the most common clinical signs include local irritation and pain. ${ }^{5,19}$ Localized pain was the major finding, but we did not identify any dermal lesions on our patients. The signs and symptoms we most often observed were cold extremities, vomiting, hypertension, sweating, agitation, and tachycardia. These findings are comparable to those of studies by Bosnak et $\mathrm{al}^{6,7}$ from the same region. Bosnak et $\mathrm{al}^{6}$ reported priapism in $72.4 \%$ patients; in contrast, we observed priapism in 1 patient. Although fever was observed less frequently in this study compared with other studies, ${ }^{12,17,20}$ clinical progression was more severe in febrile patients than in patients without fever. In particular, myocarditis was more frequently observed in patients with fever. These results can be explained by the effects of cytokines: proinflammatory cytokines cause fever and other systemic symptoms; and an imbalance between proinflammatory and anti-inflammatory mediators may be associated with mortality and morbidity. ${ }^{21}$

In our patients, myocarditis and pulmonary edema occurred less frequently than reported in other studies. $6,11,12,17$ Pulmonary edema was observed in all myocarditis cases, however, supporting the hypothesis 
that pulmonary edema results from cardiac dysfunction. ${ }^{22}$ The most common dysrhythmia was sinus tachycardia, a finding consistent with various studies. ${ }^{6,7,11,12}$ Pulseless ventricular tachycardia occurred in 2 patients. Theoretically, fatal tachycardia can be found in scorpion envenomation cases, but limited reports of this condition are available in the literature; the scorpions mentioned in those reports included the Tityus species. ${ }^{23-26}$ Bucaretchi et $\mathrm{al}^{23}$ retrospectively analyzed 1327 patients stung by scorpions in Brazil; only 1 patient had ventricular tachycardia. $^{23}$ In our results, fatal tachycardia occurred in 2 cases. Scorpion venom may affect voltage-gated sodium and potassium channels and cause myocardial ischemia. Additionally, hyperglycemia and hypokalemia can cause electrocardiographic changes. ${ }^{25}$ Both our patients who died had slightly increased glucose levels, but no electrolyte imbalance.

Agitation was the most common neurologic manifestation among in our patients, and it was generally observed in the severe group. Other investigators have suggested hypertensive encephalopathy, hypoxia, and a direct effect of the venom as the major mechanisms of agitation. $^{27}$ Mydriasis, anisocoria, and seizure are rare but can occur with severe envenomation. ${ }^{27}$ Both our patients who died had mydriasis, but only 1 of them had anisocoria and seizure.

Hyperglycemia is an established poor prognostic factor with severe envenomation; its pathogenesis is discussed in detail elsewhere. ${ }^{12}$ Our results were similar to those of other studies. Glucose levels were significantly increased in the severe group compared with the mild-moderate group. Leukocytosis is generally an expected finding after severe envenomation. Bouazisi et $\mathrm{al}^{12,28}$ reported that leukocytosis is a poor prognostic factor for outcome. In our study, leukocyte levels were markedly increased in the severe group, and on admission, their troponin I, CK-MB, and CPK levels were markedly increased. Median troponin I values peaked at 24 hours and then decreased. Two patients had initial troponin levels that were normal, even though LVEF was $25 \%$ on echocardiography; 1 of these patients died. Sofer et $\mathrm{al}^{14}$ emphasized the importance of early echocardiographic evaluation and normal troponin levels on admission.

In our study, thrombocytosis in the severe group was remarkable. It was positively correlated with leukocyte and glucose levels and was negatively correlated with LVEF. Moreover, thrombocytosis was the most predictive factor for clinical severity in multivariate analyses of laboratory parameters. Thromboglobulin secretion by platelets is a known factor involved in the pathogenesis of myocardial dysfunction. ${ }^{12}$ Although a few reports are available regarding the effect of scorpion venom on platelets, ${ }^{29}$ reactive thrombocytosis in scorpion envenomation has not been previously described. Bosnak et $\mathrm{al}^{7}$ studied thrombocyte levels but observed no difference between severe and mild-moderate envenomation cases. Mohseni et $\mathrm{al}^{30}$ reported patients stung by Hemiscorpius lepturus or A crassicauda in Iran; no difference in platelet levels was observed in those patients. Several mechanisms may explain this phenomenon. Similar to leukocytosis, interleukin-6, thrombopoietin, interleukin1 , interleukin-4, interferon $\gamma$, and tumor necrosis factor- $\alpha$ play major roles in the pathogenesis of reactive thrombocytosis. ${ }^{31}$ Moreover, there is an $\alpha$-adrenergic receptor on platelets. The release of corticosteroids and catecholamines, especially adrenaline, can induce platelets. ${ }^{32}$ Another mechanism involves the effect of hyperglycemia on platelets; this phenomenon was reported for diabetes patients with acute myocardial infarction by Oswald et al. ${ }^{32}$ Acute myocardial ischemia itself can cause reactive thrombocytosis; this phenomenon has been cited in 2 case reports in the literature. ${ }^{33}$

Our study had limitations similar to those of other retrospective studies. Also, we were unable to discover the scorpion species, and information was missing from the medical records.

\section{Conclusion}

Our epidemiological, clinical, and laboratory findings have some similarities to those of other studies. Remarkable thrombocytosis in the severe group was the most important finding of our study. Moreover, thrombocytosis was the most predictive of the laboratory parameters. Most interesting were the 2 patients with pulseless ventricular tachycardia. Finally, troponin levels in the severe group were high and peaked at 24 hours, whereas CK-MB levels were high on admission and then gradually decreased until follow-up. These results may provide illuminating data for future studies.

\section{References}

1. Cesaretli Y, Ozkan O. Scorpion stings in Turkey: epidemiological and clinical aspects between the years 1995 and 2004. Rev Inst Med Trop Sao Paulo. 2010;52:215-220.

2. Adiguzel S, Ozkan O, Inceoglu B. Epidemiological and clinical characteristics of scorpionism in children in Sanliurfa, Turkey. Toxicon. 2007;49:875-880.

3. Prendini L, Wheeler WC. Scorpion higher phylogeny and classification, taxonomic anarchy, and standards for peer review in online publishing. Cladistics. 2005;21:446-494.

4. Abroug F, ElAtrous S, Nouira S, Haguiga H, Touzi N, Bouchoucha S. Serotherapy in scorpion envenomation: a randomised controlled trial. Lancet. 1999;354:906-909.

5. Chippaux JP, Goyffon M. Epidemiology of scorpionism: a global appraisal. Acta Tropica. 2008;107:71-79. 
6. Bosnak M, Levent Yilmaz H, Ece A, et al. Severe scorpion envenomation in children: management in pediatric intensive care unit. Hum Exp Toxicol. 2009;28:721-728.

7. Bosnak M, Ece A, Yolbas I, Bosnak V, Kaplan M, Gurkan F. Scorpion sting envenomation in children in southeast Turkey. Wilderness Environ Med. 2009;20:118-124.

8. Bawaskar HS, Bawaskar PH. Scorpion sting: update. J Assoc Phys India. 2012;60:46-55.

9. Hartman ME, Cheifetz IM. Pediatric emergencies and resuscitation. In: Kliegman RM, Emerson W, eds. Nelson Textbook of Pediatrics. 19th ed. Philadelphia, PA: Elsevier/Saunders; 2011:279-295.

10. Canter CE, Simpson KP. Diagnosis and treatment of myocarditis in children in the current era. Circulation. 2014;129:115-128.

11. Bahloul M, Chabchoub I, Chaari A, et al. Scorpion envenomation among children: clinical manifestations and outcome (analysis of 685 cases). Am J Trop Med Hygiene. 2010;83:1084-1092.

12. Bouaziz M, Bahloul M, Kallel H, et al. Epidemiological, clinical characteristics and outcome of severe scorpion envenomation in South Tunisia: multivariate analysis of 951 cases. Toxicon. 2008;52:918-926.

13. Gwee MC, Nirthanan S, Khoo HE, Gopalakrishnakone P, Kini RM, Cheah LS. Autonomic effects of some scorpion venoms and toxins. Clin Exp Pharmacol Physiol. 2002;29:795-801.

14. Sofer S, Zucker N, Bilenko N, et al. The importance of early bedside echocardiography in children with scorpion envenomation. Toxicon. 2013;68:1-8.

15. Ben-Abraham R, Eschel G, Winkler E, Weinbroum AA, Barzilay Z, Paret G. Triage for Leiurus quinquestriatus scorpion envenomation in children-is routine ICU hospitalization necessary? Hum Exp Toxicol. 2000;19:663666.

16. Ulug M, Yaman Y, Yapici F, Can-Ulug N. Scorpion envenomation in children: an analysis of 99 cases. Turk $J$ Pediatr. 2012;54:119-127.

17. Mohamad IL, Elsayh KI, Mohammad HA, et al. Clinical characteristics and outcome of children stung by scorpion. Eur J Pediatr. 2014;173:815-818.

18. Osnaya-Romero N, de Jesus Medina-Hernandez T, FloresHernandez SS, Leon-Rojas G. Clinical symptoms observed in children envenomated by scorpion stings, at the children's hospital from the State of Morelos, Mexico. Toxicon. 2001;39:781-785.

19. Isbister GK, Bawaskar HS. Scorpion envenomation. N Engl J Med. 2014;371:457-463.

20. Pipelzadeh MH, Jalali A, Taraz M, Pourabbas R, Zaremirakabadi A. An epidemiological and a clinical study on scorpionism by the Iranian scorpion Hemiscorpius lepturus. Toxicon. 2007;50:984-992.

21. Petricevich VL. Scorpion venom and the inflammatory response. Mediat Inflamm. 2010;2010:903295.

22. Bahloul M, Chaari A, Dammak H, et al. Pulmonary edema following scorpion envenomation: mechanisms, clinical manifestations, diagnosis and treatment. Int $J$ Cardiol. 2013;162:86-91.

23. Bucaretchi F, Fernandes LC, Fernandes CB, et al. Clinical consequences of Tityus bahiensis and Tityus serrulatus scorpion stings in the region of Campinas, southeastern Brazil. Toxicon. 2014;89C:17-25.

24. Izquierdo LM, Rodriguez Buitrago JR. Cardiovascular dysfunction and pulmonary edema secondary to severe envenoming by Tityus pachyurus sting. Case report. Toxicon. 2012;60:603-606.

25. Miranda CH, Maio KT, Moreira HT, et al. Sustained ventricular tachycardia and cardiogenic shock due to scorpion envenomation. Case Rep Med. 2014;2014: 251870 .

26. Poon-King T. Myocarditis from scorpion stings. Br Med J. 1963;1:374-377.

27. Bahloul M, Rekik N, Chabchoub I, et al. Neurological complications secondary to severe scorpion envenomation. Med Sci Monit. 2005;11:CR196-CR202.

28. Bouaziz M, Bahloul M, Hergafi L, et al. Factors associated with pulmonary edema in severe scorpion sting patients - a multivariate analysis of 428 cases. Clin Toxicol (Phila). 2006;44:293-300.

29. Brazon J, Hughes CE, Mori J, Sevcik C, D'Suze G, Watson SP. Tityus discrepans scorpion venom activates platelets through GPVI and a novel SRC-dependent signaling pathway. Platelets. 2011;22:165-172.

30. Mohseni A, Vazirianzadeh B, Hossienzadeh M, Salehcheh M, Moradi A, Moravvej SA. The roles of some scorpions, Hemiscorpius lepturus and Androctonus crassicauda, in a scorpionism focus in Ramhormorz, southwestern Iran. J Insect Sci. 2013;13:89.

31. Sulai NH, Tefferi A. Why does my patient have thrombocytosis? Hematol Oncol Clin North Am. 2012;26:285-301, viii.

32. Oswald G, Smith C, Delamothe A, Betteridge D, Yudkin J. Raised concentrations of glucose and adrenaline and increased in vivo platelet activation after myocardial infarction. Br Heart J. 1988;59:663-671.

33. Dumrongmongcolgul N, Mankongpaisarnrung C, Sutamtewagul G, et al. Reactive thrombocytosis associated with acute myocardial infarction following STEMI with percutaneous coronary intervention. Case Rep Cardiol. 2013; 2013:707438. 